\title{
Condições de Pré-Natal de Gestantes Usuárias de Drogas Lícitas que Pariram em Maternidade de Referência em Maceió, 2013-2014
}

Prenatal Conditions of Pregnant Women Users Legal Drugs That Calved in Reference Hospital in Singapore, 2013-2014

Condiciones Prenatales de Las Mujeres Embarazadas que Lícitas Medicamentos que Parieron en la Maternidad de Referencia en Maceió, 2013-2014

Ianara Ramos de Oliveira Lemos ${ }^{1}$

Gabriela Monte Tenório Taveira ${ }^{2}$

\section{Resumo}

Objetivo: descrever as condições de prénatal de parturientes que confirmaram uso de drogas lícitas na gestação que pariram em maternidade de referência de Maceió, AL. Método: os dados foram coletados a partir dos prontuários e cartão da gestante e através de entrevista com as parturientes, mediante aprovação com TCLE. A entrevista era composta de questões que interrogavam a situação da gestacional atual, de gestações prévias, padrão de consumo de drogas e condições socioeconômicas.

Posteriormente os dados foram analisados utilizando o software Excel
2010. Não há conflito de interesse no presente estudo. Resultados: as puérperas estudadas apresentam o seguinte perfil: são menores de idade $(56,7 \%)$, provenientes da periferia da capital alagoana ou do interior do estado; com renda familiar de baixa a média; baixa escolaridade e $50 \%$ vivem em união estável não oficializada e relatam dificuldade no acesso ao sistema de saúde. $90 \%$ realizaram pré-natal em suas localidades e $10 \%$ realizaram fora da sua localidade; $52 \%$ tiveram um mínimo de 6 consultas durante a gestação; 83,3\% fizeram pelo menos um exame de imagem (USG), 66,6\% não realizaram teste para HIV e $63,3 \%$ não realizaram o

${ }^{1}$ Graduada em Medicina (UFAL). Autora correspondente: Rua Doutor Cláudio Lívio, 124, Farol, Maceió, AL, Brasil. CEP: 57055180. E-mail: ianaralemos@ hotmail.com ${ }^{2}$ Graduada em Medicina (UFAL). 
VDRL. Todas as pacientes confirmaram uso de drogas lícitas, sendo que $46,7 \%$ só usaram álcool, $20 \%$ só usaram cigarro e $33,3 \%$ fumaram e beberam durante a gravidez. Conclusão: Dentro desse contexto, é evidente a necessidade de uma ampla reestruturação no pré-natal e atenção básica como um todo, com implementação do que de fato é preconizado e modernização e atualização do sistema de Saúde para que haja de fato atenção à saúde humanizada e de qualidade.

\section{Palavras-chaves: Cuidado Pré-Natal; Preparações Farmacêuticas; Atenção Primária à Saúde.}

\section{Abstract}

Objective: To describe the prenatal mothers conditions confirmed use of licit drugs in pregnancy that calved in reference maternity Maceio, $A L$. Method: Data were collected from medical records and the pregnant woman's card and through interviews with the mothers, upon approval with IC. The interview consisted of questions that questioned the status of the current pregnancy of previous pregnancies, drug consumption patterns and socioeconomic conditions. Subsequently the data were analyzed using Excel software 2010. There is no conflict of interest in this study. Results: puerperae have the following profile: are minors (56.7\%) from the outskirts of the capital of Alagoas and in the state; with family income of low to medium; low education and $50 \%$ live in a stable relationship and no official report difficulty in accessing the health system. 90\% received prenatal care in their localities and $10 \%$ held outside their locality; $52 \%$ had at least 6 visits during pregnancy; $83.3 \%$ had at least one imaging (USG), $66.6 \%$ did not undergo testing for HIV and $63.3 \%$ did not perform VDRL. All patients confirmed use of licit drugs, and only 46.7\% used alcohol, $20 \%$ used only cigarette and $33.3 \%$ smoked and drank during pregnancy. Conclusion: In this context, it is clear the need for extensive restructuring in prenatal and primary care as a whole, with implementation than it is advocated and modernization and updating of the healthcare system so that there is in fact health care humanised and quality. 
Keywords: $\quad$ Prenatal Care;

Pharmaceutical Preparations; Primary

Health Care.

\section{Resumen}

Objetivo: Describir las condiciones madres prenatales uso confirmado de drogas lícitas en el embarazo que parieron en la maternidad de referencia Maceió, AL. Método: Se recogieron datos de los registros médicos y de la tarjeta de la mujer embarazada y mediante entrevistas con las madres, con la aprobación con IC. La entrevista consistió en preguntas que cuestionaban el estado del embarazo actual de embarazos anteriores, los patrones de consumo de drogas y las condiciones socioeconómicas.

Posteriormente se analizaron los datos utilizando el software Excel 2010. No existe ningún conflicto de interés en este estudio. Resultados: puérperas tienen el siguiente perfil: son menores de edad $(56,7 \%)$ de las afueras de la capital de Alagoas y en el estado; con un ingreso familiar de bajo a medio; bajo nivel de educación y el $50 \%$ vive en una relación estable y sin dificultad informe oficial en el acceso al sistema de salud. 90\% recibieron atención prenatal en sus localidades y $10 \%$ en manos fuera de su localidad; 52\% tenía al menos 6 visitas durante el embarazo; 83,3\% tenía al menos una imagen (USG), el $66,6 \%$ no se sometió a la prueba del Objetivo: Describir las condiciones madres prenatales uso confirmado de drogas lícitas en el embarazo que parieron en la maternidad de referencia Maceió, AL. Método: Se recogieron datos de los registros médicos y de la tarjeta de la mujer embarazada y mediante entrevistas con las madres, con la aprobación con IC. La entrevista consistió en preguntas que cuestionaban el estado del embarazo actual de embarazos anteriores, los patrones de consumo de drogas y las condiciones socioeconómicas. Posteriormente se analizaron los datos utilizando el software Excel 2010. No existe ningún conflicto de interés en este estudio. Resultados: puérperas tienen el siguiente perfil: son menores de edad $(56,7 \%)$ de las afueras de la capital de Alagoas y en el estado; con un ingreso familiar de bajo a medio; bajo nivel de educación y el $50 \%$ vive en una relación estable y sin dificultad informe oficial en el acceso al sistema de salud. 90\% recibieron atención prenatal en sus localidades y $10 \%$ en manos fuera de su localidad; $52 \%$ tenía al menos 6 
visitas durante el embarazo; 83,3\% tenía al menos una imagen (USG), el $66,6 \%$ no se sometió a la prueba del VIH y el 63,3\% no realizó VDRL. Todos los pacientes confirmaron el uso de drogas lícitas, y sólo el 46,7\% utilizan el alcohol, el $20 \%$ utiliza sólo el cigarrillo y el 33,3\% ahumado y bebieron durante el embarazo. Conclusión: En este contexto, es evidente la necesidad de una amplia reestructuración de la atención prenatal y primaria en su conjunto, con la implementación de lo que se defiende y la modernización y actualización del sistema de salud para que no haya hecho en la atención sanitaria humanizada y la calidad.

Palabras clave: Atención Prenatal; Preparaciones Farmacéuticas;

\section{Atención Primaria de Salud.}

\section{Introdução}

A gestação, o parto e o puerpério representam uma experiência humana das mais significativas para todos que dela participam. São eventos sociais que integram a vivência reprodutiva de homens e mulheres ${ }^{(1)}$. Estes são períodos de mudanças físicas e emocionais, que cada mulher vivencia de diferentes formas. Essas transformações podem gerar medos, dúvidas, angústias, fantasias ou simplesmente a curiosidade de saber o que acontece no interior de seu corpo ${ }^{(2)}$.

A atenção pré-natal e puerperal, como parte Assistência Integral à Saúde da Mulher, objetiva o acolhimento à mulher desde o início da gravidez, assegurando, no fim da gestação, o nascimento de uma criança saudável, bem como a garantia do bem-estar materno e neonatal. Para tanto, é necessária uma atenção pré-natal qualificada e humanizada, por meio da incorporação de condutas acolhedoras, sem intervenções desnecessárias; do fácil acesso a serviços de saúde, com ações que integrem todos os níveis da atenção: promoção, prevenção e assistência à saúde da gestante e do recém-nascido, desde o atendimento ambulatorial básico ao atendimento hospitalar para alto risco ${ }^{(3)}$.

O pré-natal deve ter início precoce, cobertura universal, aspecto multidisciplinar, ser realizado de forma periódica, com um mínimo de seis consultas; estar integrado às demais ações preventivas e curativas, e ser capaz de desenvolver as seguintes atribuições durante: escuta ativa da 
mulher e acompanhante, preparo à maternidade; realização de atividades educativas; estímulo ao parto normal; anamnese e exame clínico-obstétrico; prevenção e tratamento dos distúrbios nutricionais e de doenças existentes, que de alguma forma interfiram no bom andamento da gravidez; prevenção, diagnóstico precoce e tratamento de doenças próprias da gestação ou intercorrências previsíveis dela; classificação de risco gestacional e detecção de problemas; atendimento às gestantes com problemas ou comorbidades, garantindo vínculo e acesso à unidade de referência para atendimento ambulatorial e/ou hospitalar especializado; registro em prontuário e cartão da gestante, inclusive registro de intercorrências/urgências que requeiram avaliação hospitalar em situações que não necessitem de internação ${ }^{(3)}$.

$\mathrm{Na}$ avaliação dos programas de assistência ao pré-natal observa-se que muitos aspectos importantes para a saúde da gestante e do feto não são sistematicamente avaliados. A orientação no pré-natal sobre o uso de drogas de abuso, principalmente, no nosso meio, ainda é insuficiente. $\mathrm{O}$ diagnóstico ante natal e a intervenção no sentido de diminuir o abuso de drogas são a situação ideal, porém não tem sido a prática diária dos serviços de atendimento à gestante no nosso meio. Existem poucos dados nacionais referentes a gestantes usuárias de drogas, apesar do interesse em se saber efeitos clínicas e sociais sobre o binômio mãe-filho devido uso dessas substâncias.

No Brasil, as características regionais, os aspectos sociais, culturais e o nível sócio econômico, assim como o acesso aos serviços de saúde, devem influenciar no consumo de drogas pela população. O número de internações hospitalares por dependência de drogas tem aumentado consideravelmente. As idades que têm predominado estão entre 18 e 30 anos e, quando se avalia o sexo feminino, acomete principalmente mulheres em idade fértil, chamando atenção para a necessidade de intervenção governamental para esse grave problema ${ }^{(4)}$.

Um pré-natal de qualidade implica no desenvolvimento de uma gestação bem assistida, permitindo assim a detecção de fatores que possam causar prejuízos maternos e fetais. A avaliação de um pré-natal de qualidade perpassa pela análise do desenvolvimento adequado e completo do que se é instituído para o mesmo: 
desde a realização das consultas clínicas, realização de exames, atividades educativas, ao preenchimento adequado do cartão/prontuário da gestante.

\section{Método}

O estudo é do tipo transversal descritivo observacional, desenvolvido em maternidade de referência em Maceió, Alagoas, no período de 2013 a 2014, com a participação de 30 parturientes que confirmaram o uso de drogas lícitas e/ou ilícitas durante a gestação. A seleção das participantes ocorreu independentemente de raça, cor, idade, classe social ou qualquer outra variável pertinente.

Os dados foram coletados a partir dos prontuários, disponibilizados pelo hospital, e do cartão da gestante. E por meio de entrevista, com aplicação de questionário qualitativo, com a mãe usuária de droga. O TCLE - Termo de Consentimento Livre e Esclarecido - foi aplicado neste momento. No questionário constam perguntas que visam complementar os dados obtidos nos outros instrumentos, buscando informações quanto às condições socioeconômicas, escolarização, modo de vida, cuidados pré-natais, condições da gestação, condições de parto e o uso de drogas lícitas e/ou ilícitas. Os dados obtidos foram analisados utilizando o software Excel 2007.

O projeto foi aprovado pelo Comitê de Ética em Pesquisa da Universidade Federal de Alagoas, processo número 13741913.2.0000. 5013.

\section{Resultados}

As puérperas estudadas estão distribuídas nas mais diversas faixasetárias, sendo a mais nova com 14 anos e a mais velha com 42 anos. Houve número significativo de pacientes adolescentes (até 19 anos) que confirmaram usar drogas lícitas durante a gestação. $\mathrm{O}$ padrão etário das entrevistadas segue o padrão da população atendida por esta maternidade, onde se encontra maioria indiscutível de mulheres até 30 anos (80\%), destacando-se a parcela com gravidez precoce ( $<17$ anos).

Quanto ao grau de vulnerabilidade, as pacientes apresentam as seguintes características: são menores de idade $(56,7 \%)$, provenientes da periferia da capital alagoana ou do interior do estado_ 43,3\% delas são do interior de Alagoas, 
Pernambuco ou Sergipe. As demais, $56,7 \%$ são da periferia da capital de Alagoas_; com renda familiar de baixa a média; baixa escolaridade, a maioria não concluiu o ensino fundamental $\mathrm{e}$
$50 \%$ vive em união estável não oficializada e relatam dificuldade no acesso ao sistema de saúde.

Tabela 1: Distribuição das puérperas por idade.

\begin{tabular}{ccc}
\hline IDADE & $\mathrm{n}=30$ & $\%$ \\
\hline $14-17$ anos & 9 & $30 \%$ \\
$18-21$ anos & 8 & $26,7 \%$ \\
$22-25$ anos & 3 & $10 \%$ \\
$26-30$ anos & 4 & $13,3 \%$ \\
$31-34$ anos & 2 & $6,7 \%$ \\
$35-38$ anos & 3 & $10 \%$ \\
$39-42$ anos & 1 & $3,3 \%$ \\
\hline
\end{tabular}

Através dos dados coletados viuse que $90 \%$ das gestantes realizaram pré-natal em suas localidades e $10 \%$ não realizaram. Apesar de conseguirem atendimento elas relataram dificuldades na marcação de consultas e na realização de exames laboratoriais e de imagem. Com relação ao critério número de consultas, $51,8 \%$ das pacientes que fizeram pré-natal realizaram no mínimo 06 consultas, destas, $7 \%$ realizaram entre 10 a 12 consultas de pré-natal, por apresentaram intercorrências durante a gravidez; e $48,1 \%$ fizeram menos consultas do que recomenda o Ministério da Saúde. Algumas mães justificaram o baixo número de consultas no pré-natal à demora no agendamento de consultas devido a grande demanda e a falta de número adequado de profissionais na área. Há ainda o problema do deslocamento para a capital para a realização do parto, o que gera superlotação no sistema e prejuízos para o binômio materno-fetal.

Com relação aos exames disponíveis para as gestantes, o foco desta pesquisa foi a realização de ultrassonografia (USG) e sorologias, devido ao fácil acesso nos prontuários e cartão da gestante, e por serem considerados de suma importância no bom acompanhamento da gravidez. 
Nesse aspecto, 83,3\% realizaram pelo menos uma ultrassonografia durante a gravidez e apenas $16,7 \%$ delas não realizaram nenhum exame de imagem.

Daquelas que realizaram alguma USG,
$80 \%$ fizeram mais de 02 exames ultrassonográficos e $20 \%$ realizaram de 04 a 06 exames.

Tabela 2: Número de ultrassonografias realizadas pelas puérperas pesquisadas durante pré-natal.

\begin{tabular}{ccc}
\hline $\mathrm{N}^{\circ}$ DE USG & $\mathrm{N}=25$ & $\%$ \\
REALIZADAS & & \\
\hline & 5 & $20 \%$ \\
1 EXAME & 8 & $32 \%$ \\
2 EXAMES & 7 & $28 \%$ \\
3 EXAMES & 5 & $20 \%$ \\
$4-6$ EXAMES & 5
\end{tabular}

As sorologias investigadas foram:

VDRL, HIV, RUBÉOLA, CMV, TOXOPLASMOSE, HBV, HCV e HTLV. As de mais fácil acesso no sistema de saúde são o VDRL e do HIV, estas tiveram o percentual de não realização durante o pré-natal de $63,3 \%$ e $66,6 \%$, respectivamente. As sorologias menos realizadas foram para detecção de HTLV $(93,3 \%)$ e para citomegalovírus - CMV (96,6\%). 
Tabela 3: Número de sorologias realizadas-não reagentes e não realizadas pelas pesquisadas durante o pré-natal.

\begin{tabular}{llclc}
\hline SOROLOGIAS & \multicolumn{2}{l}{ REALIZADAS } & \multicolumn{2}{l}{ NÃO REALIZADAS } \\
\hline VDRL & $\mathrm{N}$ & $\%$ & $\mathrm{~N}$ & $\%$ \\
HIV & 11 & $36,6 \%$ & 19 & $63,3 \%$ \\
RUBEOLA & 10 & $33,3 \%$ & 20 & $66,6 \%$ \\
CMV & 4 & $10 \%$ & 26 & $86,6 \%$ \\
TOXOPLASMOSE & 1 & $3,3 \%$ & 29 & $96,6 \%$ \\
HBV & 5 & $13,3 \%$ & 25 & $83,3 \%$ \\
HCV & 5 & $10 \%$ & 25 & $83,3 \%$ \\
HTLV & 5 & $10 \%$ & 25 & $83,3 \%$ \\
\hline
\end{tabular}

No grupo observado, $63,3 \%$ não realizou o VDRL, teste que pesquisa infecção pela bactéria Treponema pallidum, causadora da sífilis. Desta forma, não se sabe fidedignamente quantas gestantes estão infectadas e então não se pode fazer prevenção na transmissão vertical desta doença, o que realmente preocupa já que a sífilis congênita é a infecção congênita mais comum no Brasil ${ }^{(9)}$.

Nenhuma teve sorologia reagente notificada através do prontuário ou confirmou verbalmente esta condição. A maioria não realizou sorologias antes do parto ou os instrumentos que foram utilizados para a coleta desta informação, prontuário ou cartão da gestante, não foram corretamente preenchidos. Já as pacientes que realizaram algumas destas sorologias tiveram resultados sempre nãoreagentes.
No tocante ao diagnóstico e tratamento das síndromes hipertensivas na gravidez, principal causa de morte materna, apenas 01 gestante relatou fazer uso de metildopa por aumento da pressão arterial durante a gestação, sem história prévia de hipertensão arterial sistêmica.

Houve dificuldades para encontrar dados que confirmassem o estado vacinal da gestante (cartão/prontuário), assim, as informações obtidas foram colhidas verbalmente junto as pacientes. Destas, $46,7 \%$ possuem cartão vacinal atualizado, 43,3\% não sabe a real condição vacinal, (nada consta no prontuário ou no cartão da gestante). Apenas $10 \%$ não estão com as vacinas atualizadas.

Quanto ao padrão de consumo de drogas lícitas, o gráfico 2 mostra que $46,7 \%$ das puérperas afirmaram ter feito uso de apenas bebida alcoólica em 
algum momento da gestação, sendo a cerveja a bebida mais consumida $(84,7 \%)$, seguida da vodca $(11,5 \%)$. $20 \%$ fizeram uso de somente tabaco, representado principalmente na forma de cigarro, e $33,3 \%$ fizeram uso concomitante de álcool e tabaco, o que aumenta ainda mais o risco de problemas para o feto e a mãe, e de intercorrências gestacionais por efeito sinérgico entre essas substâncias.

$\mathrm{O}$ conhecimento das gestantes a respeito dos malefícios do uso de drogas para o para ela e o feto é limitado, uma parcela importante relata saber que o uso destas substâncias faz mal para a saúde, mas não sabem informar o porquê ou dar outras informações (80\%). Todas as gestantes tiveram o mesmo discurso com relação ao suporte de informações no pré-natal: afirmam que poucas orientações foram dadas durante as consultas e que foram raras as vezes que algum profissional da equipe se interessou em saber se elas usavam drogas. $\mathrm{O}$ conhecimento prévio sobre o assunto foi advindo dos meios de comunicação ou de conversas com amigos/familiares.

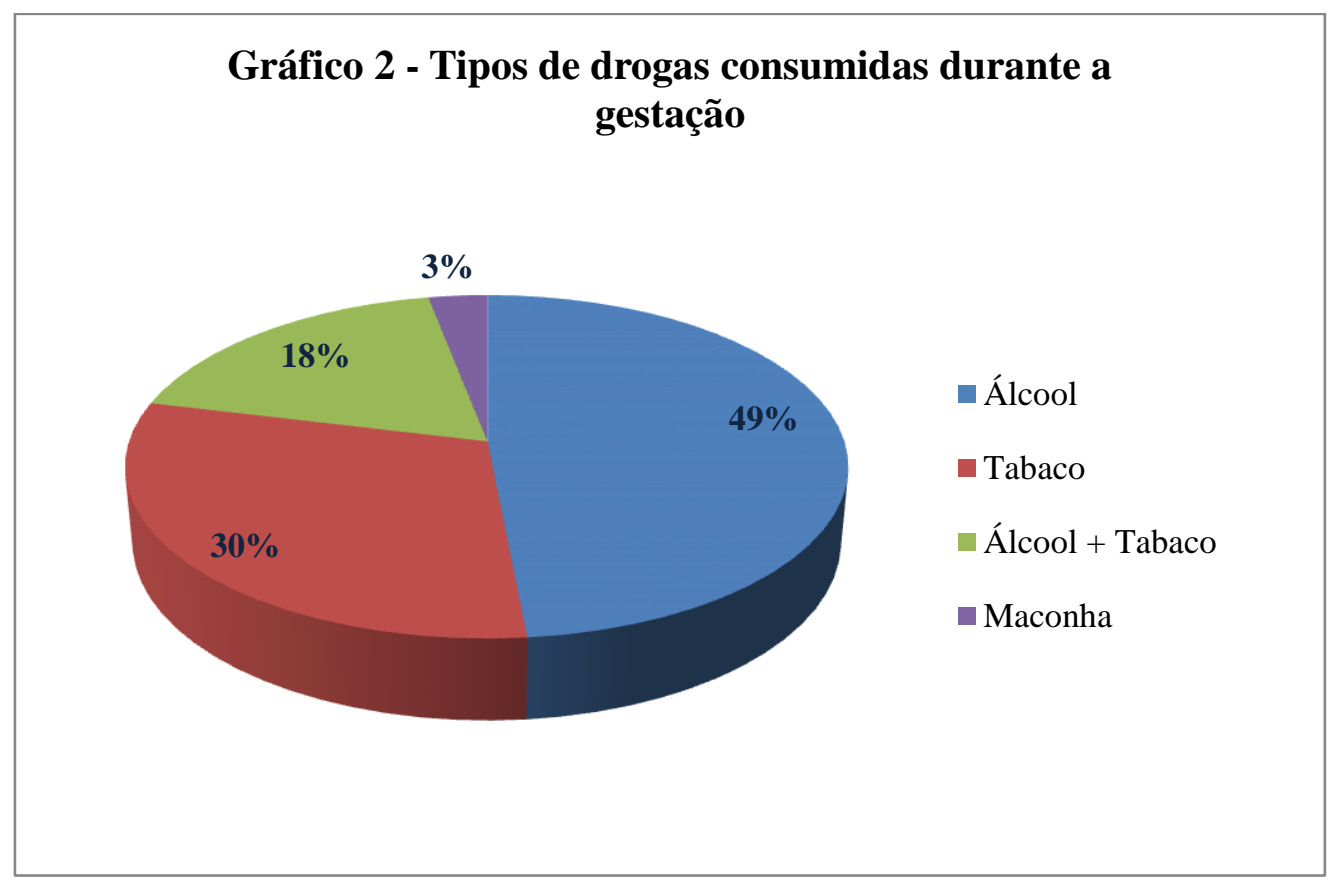

\section{Discussão}

A faixa etária materna não deve ser encarada como um fator meramente biológico que, isoladamente, pode acarretar complicações para a mãe e seu filho. Destaca-se que mais importante do que a idade, seriam as condições de 
vida e saúde das gestantes, principalmente, a qualidade da assistência obstétrica no pré-natal e no parto. A idade materna menor que $17 \mathrm{e}$ maior que 35 anos representa um fator de risco importante na $\operatorname{gravidez}^{(5)}$. A literatura aponta que os problemas mais frequentes encontrados nas jovens mães são a maior incidência de doença hipertensiva e anemia, menor ganho de peso, além de complicações no parto, com consequente aumento da mortalidade materna. Com relação aos problemas do recém-nascido, pode-se mencionar o baixo peso ao nascer, prematuridade e anóxia ${ }^{(5)}$.

Em virtude da pouca idade materna pode-se refletir sobre as influências que esta adolescente sofre durante a gravidez: imaturidade materna, baixa escolaridade, baixa renda familiar, estado nutricional prejudicado, instabilidade emocional e maior influência de maus hábitos (drogas, violência).

A maior parte das gestantes estudadas faz parte deste grupo socioeconômico de risco: são mães jovens, solteiras ou com relação conjugal instável, com renda familiar de baixa a média e baixa escolaridade. Toda essa conjuntura favorece complicações para o binômio materno- fetal, como já descrito.

No Brasil, o acesso ao pré-natal como condição primária para a assistência apresenta ainda importantes diferenças por região, residência e escolaridade. A cobertura da assistência pré-natal no Brasil ainda é baixa e as desigualdades no uso desta assistência persistem. O percentual de mulheres da zona rural que não realizam o pré-natal é alto. Havendo também grande diferença na cobertura segundo regiões geográficas $^{(6)}$.

Assim como descreve a literatura, o acesso à atenção primária, e consequentemente ao pré-natal, é precário; e quando se tem acesso há outras dificuldades que influenciam no bom desenvolvimento deste serviço, como a marcação e realização de exames, a agilidade na disponibilidade dos resultados dos mesmos, falta de recursos no município de residência, sendo necessário descolamento para áreas metropolitanas, todos esses fatores favorecem falha na detecção e intervenção precoces de doenças e agravos.

Segundo Brito $^{(7)}$, no Brasil, o Programa de Humanização no Pré-natal e Nascimento (PHPN) estabelece alguns critérios para a realização de um bom pré-natal utilizando um mínimo de 
procedimentos considerados essenciais e que pudessem ser implementados na grande maioria dos municípios brasileiros. São eles: número de consultas obrigatórias, mínimo de seis consultas de pré-natal, sendo uma no primeiro, duas no segundo e três no terceiro trimestre da gestação, mas a primeira consulta deve ocorrer até o $4^{\circ}$ mês de gestação. Acesso a uma consulta no puerpério, até 42 dias após o nascimento.

A realização de exames complementares, imprescindível na boa conduta do pré-natal deve ser garantida. Os principais exames são: $\mathrm{ABO}-\mathrm{Rh}$; VDRL, um exame na primeira consulta e outro próximo à trigésima semana de gestação; exame de urina, um exame na primeira consulta e outro próximo à trigésima semana de gestação; glicemia de jejum, um exame na primeira consulta e outro próximo à trigésima semana de gestação; hemoglobina/hematócrito; teste de $\operatorname{HIV}^{(7)}$.

Além disso, deve-se proporcionar vacinação antitetânica até a dose imunizante (segunda) do esquema recomendado, ou dose de reforço em mulheres já imunizadas. Bem como, elaboração de atividades educativas. Classificação de risco gestacional a ser realizada na primeira consulta e nas subsequentes, o que torna necessário a consulta com profissional médico além da enfermeira. Garantindo vínculo e acesso à unidade de referência às gestantes classificadas como de alto risco, assim como o atendimento ambulatorial e/ou hospitalar ${ }^{(7)}$.

A realização da USG no $1^{\circ}$ trimestre permite melhor acompanhamento da idade gestacional, detecção precoce de gestações gemelares e de fetos portadores de anomalias. Não existe critério obrigatório para realização deste exame, mas normalmente recomenda-se pelo menos uma USG durante a gestação e idealmente uma USG a cada trimestre ${ }^{(8)}$.

$\mathrm{O}$ dado referente à quantidade de USG revela o quanto a população preconiza este exame aos demais. Pelo que se constatou, existe um desejo, quase que velado, de olhar o feto por imagem, mesmo que não se possa ver o sexo, apenas o fato de apreciar e constatar a saúde deste já tranquiliza a gestante e sua família.

As sorologias também fazem parte da rotina do pré-natal, detectam infecções e orientam quanto à prevenção/tratamento. Ao contrário do que ocorre com a realização da USG, que apresenta alta taxa de realização do 
exame, no grupo estudado, a taxa de realização dessas sorologias é baixa. Isto pode se dar tanto pela falta de conhecimento dessas parturientes da importância desses exames para ela e para o feto, como pela dificuldade de acesso a eles e demora na entrega dos resultados, sendo alguns entregues após o parto, não tendo mais utilidade na prevenção dependendo do exame. Sendo assim, a realização de exames laboratoriais na assistência pré-natal é imensamente inapropriada, perdendo-se oportunidade de prevenção de transmissão vertical e tratamento.

A qualidade na assistência prénatal pode ser analisada sob a perspectiva de diversos fatores $\mathrm{e}$ índices. Segundo Brito ${ }^{(7)}$, três principais indicadores confirmam problemas na assistência pré-natal no Brasil. Estes são: a alta incidência de sífilis congênita (24/1000 nascidos vivos no SUS), a Doença Hipertensiva Específica da Gravidez (DHEG) e a baixa cobertura vacinal contra o tétano em gestantes.

A sífilis pode ter diagnóstico e tratamento totalmente conduzido na assistência pré-natal. $\mathrm{O}$ exame diagnóstico desta patologia é uma das sorologias mais pedidas na Atenção Básica. Assim altos índices desta patologia indicam deficiência na assistência.

A realização de sorologia para detecção de sífilis, VDRL, teve baixa cobertura no grupo estudado, o que seguramente implica deficiência na atenção primária. Perde-se aqui, mais uma vez, a chance de intervenção e interrupção da transmissão vertical.

A DHEG é a principal causa de óbitos maternos, podendo ser diagnosticada pela mensuração sistemática da pressão arterial associada à identificação de sinais e sintomas clínicos, além de exames complementares. A facilidade do diagnóstico demonstra que, se ocorrer alta mortalidade com esta causa base, o pré-natal não foi bem conduzido.

Segundo Santos $^{(10)}$, as vacinas recomendadas para as gestantes são: tétano e difteria; Hepatite B; sarampo, caxumba e rubéola; febre amarela e a Influenza. A vacinação em gestantes tem como principal objetivo a proteção da mulher grávida, do feto, recémnascido e/ou lactente. A boa cobertura vacinal garante diminuição de doenças e complicações da gestação e favorece a proteção do filho, que ainda possui sistema imunológico imaturo ${ }^{(11)}$.

A falta de notificação no cartão da gestante da situação vacinal da mesma dificulta intervenção e boa condução do 
pré-natal, gestação e puerpério. O não preenchimento adequado e completo do cartão da gestante, quesito obrigatório do pré-natal, permite que haja falhas na condução do pré-natal, parto e puerpério; pois, perde-se a referência da situação gestacional atual e prévias, quando existente, o que essencial na tomada de decisão da conduta clínica.

Quanto ao padrão de consumo de rogas lícitas este segue o que é observado na literatura. Matta et $\mathrm{al}^{(12)}$, em estudo com amostra composta por 137 gestantes entre 18 e 43 anos durante a gestação, encontrou que $14,6 \%$ das gestantes afirmaram terem consumido alguma quantidade de bebidas alcoólicas, sendo a cerveja a bebida mais consumida $(52,6 \%$ das gestantes revelaram consumir bebidas alcoólicas). Quanto ao consumo de cigarros durante a gestação, $17,5 \%$ das gestantes afirmaram tê-los consumido.

A exposição intrauterina ao álcool, resultante do consumo de bebidas alcoólicas pela gestante, leva a vários efeitos deletérios ao embrião e ao feto. Estes, agrupados sobre o termo espectro desordens fetais alcoólicas (fetal alcohol spectrum disorders FASD) incluem alterações físicas, mentais, comportamentais e/ou de aprendizado. Com possibilidade dessas alterações se perpetuarem por toda a vida, e alto risco para os indivíduos afetados se tornarem dependentes de álcool e de outras drogas, terem problemas mentais, dificuldades escolares/trabalho, comportamento sexual inapropriado e problemas com a justiça $^{(13)}$.

O consumo de tabaco nos primeiros meses de gestação pode implicar em abortamento, já o consumo durante toda a gestação pode levar também a problemas como: descolamento prematuro de placenta, restrição do crescimento fetal intrautero, rotura prematura de membranas ovulares, trabalho de parto prematuro, recém-nascido prematuro e/ou baixo peso $^{(14)}$. Os efeitos destas drogas de abuso podem ser percebidos ainda durante a gestação ou podem ter sua influência detectada só em outras fases da vida, mas o efeito negativo sempre vai ser um risco à saúde.

\section{Conclusão}

É grande a deficiência na Atenção à saúde da gestante e do concepto, uma vez que o pré-natal não vem sendo realizado de maneira adequada, seja por falta de recursos financeiros, materiais e humanos ou por questões técnicas; com 
dificuldades que perpassam os três níveis de atenção à saúde: promoção, prevenção e reabilitação de agravos à saúde. A amostra estudada não recebeu, na grande maioria, informações esclarecedoras sobre os malefícios do uso de drogas, de qualquer tipo, por parte das equipes de saúde, refletindo a falha das ações de educação em saúde.

Sugere-se que medidas para que haja um melhor acolhimento e qualidade no pré-natal devam ser tomadas, como: capacitação dos profissionais de saúde para abordar os temas de educação em saúde propostos pelo programa de pré-natal, bem como reciclagem continuada dos profissionais de saúde com temas inerentes ao prénatal e Atenção Básica; estabelecimento de protocolos/fiscalização quanto ao preenchimento de cartão de gestante e prontuário; busca da compreensão por parte das gestantes da importância dos exames que são realizados durante o pré-natal e importância do pré-natal como um todo; estímulo ao Controle Social, com esclarecimentos a cerca dos direitos das gestantes e dos cidadãos como um todo com relação à qualidade de assistência a saúde, para que assim possam exigi-los.

Faz-se necessário também a reestruturação do serviço de atenção primária, para que este consiga cumprir o que lhe é preconizado, garantindo um mínimo de atendimentos, exames e medicamentos que fazem parte do diaa-dia do serviço; para mudar a realidade de má prestação de serviço em saúde que afeta drasticamente a vida de todos os brasileiros.

\section{Referências}

1. Brasil. Ministério da Saúde. Secretaria de Políticas de Saúde, Área Técnica da Mulher. Parto, aborto e puerpério: assistência humanizada à mulher - Manual Técnico. Brasília: MS; 2001. Disponível em: http://bvsms.sau de.gov.br/bvs/publicacoes/cd04_13.pdf. Acesso em: 16 de abril 2015.

2. Brasil. Ministério da Saúde. Secretaria de Políticas de Saúde. Assistência Pré-natal: Manual técnico. [livro online] Brasília: MS; 2000. Disponível em: http://bvsms.saude.gov .br/bvs/publicacoes/cd04_11.pdf.

Acesso em: 16 de abril 2015.

3. Brasil, Ministério da Saúde. Secretaria de Atenção à Saúde, Área Técnica da Mulher. Pré-natal e Puerpério: atenção qualificada e humanizada - manual técnico. Brasília: MS; 2006. Disponível em: http://bvsms.saude.gov.br/bvs/publicaco es/manual_pre_natal_puerperio_3ed.pdf . Acesso em: 16 de abril 2015.

4. Zullini. MTC. et al. Drogas de abuso na gestação: as orientações no pré-natal são suficientes? Revista Pediatria. 1998;20(4): 316-22. 
5. Ximenes FMA et al. A influência da idade materna sobre as condições perinatais. RBPS 2004; 17(2): 56-60.

6. Coimbra LC et al. Fatores associados à inadequação do uso da assistência prénatal. Rev Saúde Pública. 2003; 37(4): 456-62.

7. Brito $\mathrm{AO}$ et al. Diagnóstico situacional da assistência pré-natal pelo Programa Saúde da Família no município de Corinto, Minas Gerais. Rev Bras Med Fam e Com. 2008.

8. Alencar Jr CA. Projeto Diretrizes. Federação Brasileira das Sociedades de Ginecologia e Obstetrícia. Assistência Pré-Natal; 2001. Disponível em: http://www.projetodiretrizes.org.br/proj eto_diretrizes/081.pdf. Acesso em: 02 junho 2016.

9. Guinsburg R, Santos AMN. Critérios diagnósticos e tratamento da sífilis congênita. Documento Científico Departamento de Neonatologia Sociedade Brasileira de Pediatria. São Paulo; 2010. Disponível em: http://www.sbp.com.br/pdfs/tratamento _sifilis.pdf. Acesso em: 20 de abril 2015.

10. Santos. ZMS. et al. Vacinação - o que o usuário sabe? RBPS. 2005; 1(18):24-30.

11. Louzeiro EM et al. A importância da vacinação em gestantes: uma revisão sistemática da literatura no período de 2003 a 2012. R. Interd. 2014; 7(1): 193-03.

12. Coimbra LC et al. Fatores associados à inadequação do uso da assistência pré-natal. Rev Saúde Pública. 2003; 37(4): 456-62.
13. Coimbra LC et al. Fatores associados à inadequação do uso da assistência pré-natal. Rev Saúde Pública. 2003; 37(4): 456-62.

14. Coimbra LC et al. Fatores associados à inadequação do uso da assistência pré-natal. Rev Saúde Pública. 2003; 37(4): 456-62. 